\title{
Multiscale Modeling of Astrophysical Jets
}

\author{
James H. Beall ${ }^{1,2,3}$, John Guillory* 3 , David V. Rose ${ }^{4}$, Michael T. Wolff ${ }^{2}$ \\ ${ }^{1}$ St. Johns College, Annapolis, MD \\ ${ }^{2}$ Space Sciences Division, Naval Research Laboratory, Washington, DC; \\ ${ }^{3}$ College of Sciences, George Mason University, Fairfax, VA; \\ ${ }^{4}$ Voss Scientific, Albuquerque, NM; \\ Corresponding author: beall@sjc.edu
}

\begin{abstract}
We are developing the capability for a multi-scale code to model the energy deposition rate and momentum transfer rate of an astrophysical jet which generates strong plasma turbulence in its interaction with the ambient medium through which it propagates. We start with a highly parallelized version of the VH-1 Hydrodynamics Code (Coella and Wood 1984, and Saxton, et al., 2005). We are also considering the PLUTO code (Mignone et al. 2007) to model the jet in the magnetohydrodynamic (MHD) and relativistic, magnetohydrodynamic (RMHD) regimes. Particle-in-Cell approaches are also being used to benchmark a wave-population models of the two-stream instability and associated plasma processes in order to determine energy deposition and momentum transfer rates for these modes of jet-ambient medium interactions.

We show some elements of the modeling of these jets in this paper, including energy loss and heating via plasma processes, and large scale hydrodynamic and relativistic hydrodynamic simulations. A preliminary simulation of a jet from the galactic center region is used to lend credence to the jet as the source of the so-called the Fermi Bubble (see, e.g., Su, M., \& Finkbeiner, D. P., 2012)

*It is with great sorrow that we acknowledge the loss of our colleague and friend of more than thirty years, Dr. John Ural Guillory, to his battle with cancer.
\end{abstract}

Keywords: jets - active galaxies - blazars - intracluster medium - non-linear dynamics - plasma astrophysics.

\section{Introduction}

Recent high-resolution (VLBA) observations of astrophysical jets (see, e.g., Lister et al. 2009) reveal complex structures apparently caused by ejecta from the central engine as they interacts with both surrounding interstellar material such as Broad-Line Region (BLR) and Narrow-Line Region (NRL) clouds, and ejecta from prior episodes of activity. A particularly trenchant example of these complex interactions is also shown by the galactic microquasar, Sco X-1 (Fomalhaut, Geldzahler, and Bradshaw, 2001).

Such observations can be used to inform models of the jet-ambient-medium interactions. Based on an analysis of these data, we posit that a significant part of the observed phenomena come from the interaction of the ejecta with prior ejecta as well as interstellar material.

\section{Scales of Jet Interactions with the Ambient Medium}

Large scale hydrodynamic simulations of the interaction of astrophysical jets with the ambient medium through which they propagate can be used to illuminate a number of interesting consequences of the jets' presence. These include acceleration and entrainment of the ambient medium, the effects of shock structures on star formation rates, and other effects originating from ram pressure and turbulence generated by the jet (see, e.g., Basson and Alexander, 2002; Zanni et al. 2005; and Krause and Camenzind 2003; Perucho, et al. 2012). We have presented results for large scale hydrodynamic simulations and initial relativistic hydrodynamic simulations in previous works (Beall et al., 1999, 2003, 2006).

As noted in those papers, magneto-hydrodynamic (MHD) and (RMHD) simulations neglect important species of physics: the microscopic interactions that occur because of the effects of particle-particle interactions and the interactions of particles with the collective effects that accompany a fully or partially ionized ambient medium (i.e. a plasma). While the physical processes (including plasma processes) in the ambient medium can be modeled in small regions by PIC (Particle-in-Cell) codes for some parameter ranges, simulations of the larger astrophysical jet structure with such PIC codes are not possible with current or foresee- 
able computer systems. For this reason, we have modeled these plasma processes in the astrophysical regime by means of a system of coupled differential equations which give the wave populations generated by the interaction of the astrophysical jet with the ambient medium through which it propagates. A detailed discussion of these efforts can be found, variously, in Scott et al. (1980), Rose et al. (1984), Rose et al. (1987), Beall (1990), and Beall et al. (2003). The scales of these interactions range from kilometers to kiloparsecs.

\subsection{Energy loss, energy deposition rate, and momentum transfer from plasma processes}

The system of equations used to determine the normalized wave energy densities is very stiff. Scott et al. (1980) estimated the equilibrium solution of this system of equations for heating of clusters of galaxies, and Rose et al. (1984) and Beall (1990) showed dynamical solutions that confirmed the stability of the equilibrium solutions. Solving the system of equations yields a time-dependent set of normalized wave energies (i.e., the ratio of the wave energy divided by the thermal energy of the plasma) that are generated as a result of jets interaction with the ambient medium. These solutions can yield an energy deposition rate $(d E / d t)$, an energy deposition length $(\mathrm{dE} / \mathrm{dx})$, and ultimately, a momentum transfer rate $\left(d p / d t\left(1 / v_{b}\right) *(d E / d t)\right.$ that can be used to estimate the effects of plasma processes on the hydrodynamic evolution of the jet.

For this part of the analysis, we suppose that a portion of the jet is composed relativistic particles of either $e^{ \pm}, p-e^{-}$, or more generally, a charge-neutral, hadron- $e^{-}$jet, with a significantly lower density than the ambient medium. The primary energy loss mechanism for the electron-positron jet is via plasma processes, as Beall (1990) notes. Kundt $(1987,1999)$ also discusses the propagation of electron-positron jets.

Beall et al., (2006) illustrate two possible solutions for the system of coupled differential equations that model this mode of the jet-ambient medium interaction: a damped oscillatory and an oscillatory solution. The Landau damping rate for the two-temperature thermal distribution of the ambient medium is used for these solutions.

The average energy deposition rate, $\left\langle d\left(\alpha \epsilon_{1}\right) / d t\right\rangle$, of the jet energy into the ambient medium via plasma processes can be calculated as $\left\langle d\left(\alpha \epsilon_{1}\right) / d t\right\rangle=n_{p} k T<$ $W>\left(\Gamma_{1} / \omega_{p}\right) \omega_{p}$ ergs $\mathrm{cm}^{-3} \mathrm{~s}^{-1}$, where k is Boltzmann's constant, $\mathrm{T}$ is the plasma temperature, $\langle W\rangle$ is the average (or equilibrium) normalized wave energy density obtained from the wave population code, $\Gamma_{1}$ is the initial growth rate of the two-stream instability, and $\alpha$ is a factor that corrects for the simultane- ous transfer of resonant wave energy into nonresonant and ion-acoustic waves. The energy loss scale length, $d E_{\text {plasma }} / d x=-\left(1 / n_{b} v_{b}\right)\left(d \alpha \epsilon_{1} / d t\right)$, can be obtained by determining the change in $\gamma$ of a factor of 2 with the integration $\int d \gamma=-\int\left[d\left(\alpha \epsilon_{1}\right) / d t\right] d l /\left(v_{b} n_{b} m^{\prime} c^{2}\right)$ as shown in Rose et al., 1978 and Beall 1990, where $m^{\prime}$ is the mass of the beam particle. Thus, $L_{p}=\left((1 / 2) \gamma c n_{b} m c^{2}\right) /\left(d \alpha \epsilon_{1} / d t\right) \mathrm{cm}$ is the characteristic propagation length for collisionless losses for an electron or electron-positron jet, where $d \alpha \epsilon_{1} / d t$ is the normalized energy deposition rate (in units of thermal energy) from the plasma waves into the ambient plasma. In many astrophysical cases, this is the dominant energy loss mechanism. We can therefore model the energy deposition rate $(\mathrm{dE} / \mathrm{dt})$ and the energy loss per unit length $(\mathrm{dE} / \mathrm{dx})$, and ultimately the momentum loss per unit length $(\mathrm{dp} / \mathrm{dx})$ due to plasma processes.

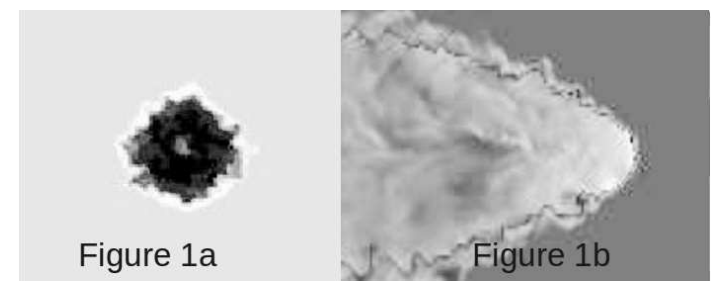

Figure 1: Simulation using a highly-parallelized version of the VH-1 hydrodynamics code. The figure shows the transverse cross-section (Figure 1a) and the detail of the jet head in the $\mathrm{y}-\mathrm{z}$ cross-section (Figure 1b) for a fully 3-dimensional hydrodynamic simulation of jet with $v=1.5 \times 10^{9} \mathrm{~cm} / \mathrm{sec}$. The cross section at $z=300$ for a jet with $v=1.0 \times 10^{1} 0 \mathrm{~cm} / \mathrm{s}$. The cross section represents approximately 2 kiloparsecs from the jet origin. We have used the $512^{3}$ simulation in this representation for ease of presentation, given the size and difficulty of displaying the $2048^{3}$ data. Note the well-developed Rayleigh-Taylor instabilities at the jet-ambient medium boundary in Figure 1a.

Beall, Guillory, and Rose (2009) have compared the results of a PIC code simulation of an electronpositron jet propagating through an ambient medium of an electron-proton plasma with the solutions obtained by the wave population model code, and have found good agreement between the two results (see Figure 1 from that paper). At the same time, that paper demonstrates that the ambient medium is heated and entrained into the jet. That analysis also shows that a relativistic, low-density jet can interpenetrate an ambient gas or plasma.

Initially, and for a significant fraction of its propagation length, the principal energy loss mechanisms for such a jet interacting with the ambient medium is via plasma processes (Rose et al. 1984, Beall 1990). 
As part of our research into the micro-physics of the interaction of jets with an ambient medium, we continue to investigate the transfer of momentum from the jet, and expect to present these results shortly. In order to proceed to a more detailed analysis of the issue of momentum transfer, we have used modern PIC code simulations to study the dynamics of caviton formation, and have confirmed the work of Robinson and Newman (1990) in terms of the cavitons' formation, evolution, and collapse.

\subsection{Results of hydrodynamical calculations}

Figure 1 shows two views of a simulation for an astrophysical jet for parameters appropriate to a Seyfert galaxy, using a highly-parallelized version of the VH1 hydrodynamics code. The figure shows the transverse cross-section (Figure 1a) and the detail of the jet head in the $y-z$ cross-section (Figure 1b) for a fully 3-dimensional hydrodynamic simulation of jet with $v=1.5 \times 10^{9} \mathrm{~cm} / \mathrm{sec}$. The cross section at $z=300$ for a jet with $v=1.0 x 10^{1} 0 \mathrm{~cm} / \mathrm{s}$. The cross section occurs at approximately 2 kiloparsecs from the jet origin.

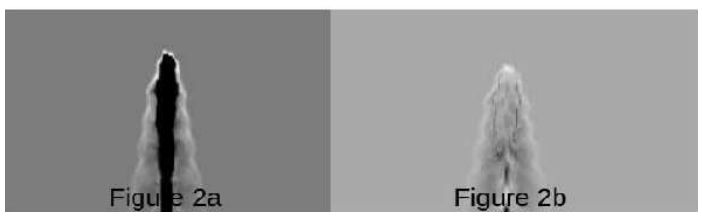

Figure 2: Simulation using the VH-1 code of a supersonic jet with parameters appropriate to the Galactic Center region. In this simulation, also, we use the VH-1 code for a supersonic jet in the $\mathrm{x}-\mathrm{y}$ pressure cross section (1a) and with an $x-y$ density cross section (1b) for a supersonic jet with $v=2.0 \times 10^{8} \mathrm{~cm} / \mathrm{s}$. Such a jet originating from the supermassive black hole at the center of the Milky Way could account for the production of the gamma-ray structure known as the Galactic Center Bubble found by the Fermi satellite. The vertical scale of the jet represents approximately 2 kiloparsecs from the jet origin. We have used the $512^{3}$ simulation in this representation for ease of presentation, given the size and difficulty of displaying the $2048^{3}$ data

Figure 2 shows a simulation of a supersonic jet for parameters appropriate to the Galactic Centre region. In this simulation, also, we use the VH-1 code for a supersonic jet in the x-y pressure cross section (1a) and with an $\mathrm{x}-\mathrm{y}$ density cross section (1b) for a supersonic jet with $v=2.0 \times 10^{8} \mathrm{~cm} / \mathrm{s}$. Such a jet originating from the supermassive black hole at the center of the Milky Way could account for the production of the gamma-ray structure known as the Galactic Center Bubble found by the Fermi satellite. The vertical scale of the jet in Figure 2a represents approximately 2 kiloparsecs from the jet origin. We have used the $512^{3}$ simulation in this representation for ease of presentation. The detail of the jet-head structure (Figure 2b) shows complex, transverse shock structures at the leading edge of the jet. The simulations we have run using $2048^{3}$ cells confirm these features.

\section{Concluding Remarks}

The effects of collective and particle processes, including plasma effects, can have observational consequences. Beall (1990) has noted that plasma processes can slow the jets rapidly, and Beall and Bednarek (1999) have shown that these effects can truncate the low-energy portion of the $\gamma$-rays spectrum (see their Figure 3), A similar effect will occur for particle-particle productions of neutrinos, pions, and (perhaps) neutrons. This could also reduce the expected neutrino flux from AGN. The presence of plasma processes in jets can also greatly enhance line radiation species by generating high-energy tails on the Maxwell-Boltzmann distribution of the ambient medium, thus abrogating the assumption of thermal equilibrium.

An analytical calculation of the boost in energy of the electrons in the ambient medium to produce such a high energy tail, with $E_{\text {het }} \sim 30-100 \mathrm{kT}$, is confirmed by PIC-code simulations. Aside from altering the Landau damping rate, such a high-energy tail can greatly enhance line radiation over that expected for a thermal equilibrium calculation (see Beall et al. 2006, and Beall, Guillory, and Rose (1999) for a detailed discussion).

We are in the process of setting up runs for the PLUTO code (Mignone et al. 2007) in order to benchmark its results with our results of the VH-1 code in appropriate parameter ranges.

\section{Acknowledgement}

JHB and MTW gratefully acknowledge the support of the Office of Naval Research for this project. Thanks also to colleagues at various institutions for their continued interest and collaboration, including Kinwah Wu, Curtis Saxton, S. Schindler and W. Kapferer, and S. Colafrancesco.

\section{References}

[1] Basson, J. F., and Alexander, P., 2002, The longterm effect of radio sources on the intracluster medium, MNRAS, 339, 353. 
[2] Beall, J. H. et al., 1978, Radio and x-ray variability of the nucleus of Centaurus A (NGC 5128), Ap.J., 219, 836. doi:10.1086/155845

[3] Beall, J. H. and Rose, W. K., 1981, On the physical environment in the nucleus of Centaurus A(NGC 5128), Ap.J., 238, 539 (also see Erratum, 1981, Ap.J., 248, 883).

[4] Beall, J. H. and Bednarek, W., 1999, On the hadronic beam model for gamma-ray production in blazars, Ap.J., 510, 188.

[5] Beall, J. H., Guillory, J., and Rose, D. V., 1999, Observational signatures in jet-cloud interactions, Mem. S. A. It., 70, 1235.

[6] Beall, J. H., Guillory, J., and Rose, D. V., 2003, AGN jet interactions with the intracluster medium, C.J.A.A., 3(Suppl.), 137.

[7] Beall, J. H., Guillory, J., Rose, D. V., Schindler, S., Colafrancesco, S., 2006, Energetics of jet interactions with the intracluster medium, C.J.A.A., 6(Suppl. 1), 283.

[8] Beall J. H., 1990, Energy loss mechanisms for fast particles in an ambient medium, in Physical Processes in Hot Cosmic Plasmas, W. Brinkmann, A. C. Fabian, and F. Giobannelli, eds. (Kluwer Academic, London), p. 341. doi:10.1007/978-94-009-0545-0_20

[9] Colella, P., and Woodward, P. R. 1984, The piecewise parabolic method (PPM) for gasdynamical simulations, J. Comp. Phys., 54, 174. doi:10.1016/0021-9991(84)90143-8

[10] Fomalont, E. B., Geldzahler, B. J., and Bradshaw, C. F., 2001, Scorpius X-1: the evolution and nature of the twin compact radio lobes, Ap.J., 558, 283. doi:10.1086/322479

[11] Kaplan, S. A., and Tsytovich, V. N., 1973 Plasma Astrophysics, (Pergamon Press, Oxford).

[12] Krause, M., and Camenzind, M., 2003, Paarameters for very light jets of $\mathrm{cD}$ galaxies, New Astron. Rev., 47, 573. doi:10.1016/S1387-6473(03)00096-4

[13] Kundt, W., 1987, The sources and their models, in Astrophysical Jets and Their Engines, ed. W. Kundt (Reidel, Dordrecht, Netherlands), p. 1.

[14] Kundt, W. 1999, SS 433, Mem. S. A. It., 70, 1097.

[15] Lister, M. L. et al., 2009, Astronom. J., 137, 3718. doi:10.1088/0004-6256/137/3/3718

[16] Mignone, A. et al., 2007, Ap.J. Suppl., 170, 228.
[17] Newman, D. L., Winglee, R. M., Robinson, P. A., Glanz, J., and Goldman, M. V., 1990, Simulation of the collapse and dissipation of Langmuir wave packets, Phys. Fluids B, 2, 2600. doi:10.1063/1.859385

[18] Perucho, M., 2012, Jets in high-mass microquasars, Mem. S. A. It., 83, 297.

[19] Rose, W. K., Guillory, J., Beall, J. H., and Kainer, S., 1984, The interaction of relativistic chargedparticle beams with interstellar clouds, Ap.J., 280, 550.

[20] Rose, W. K., Beall, J. H., Guillory, J., and Kainer, S., 1987, Radiation from relativistic beams interacting with interstellar gas, Ap.J., 314, 95. doi:10.1086/165042

[21] Saxton, C. J., Bicknell, G. V., Sutherland, R. S., and Midgley S., 2005, Interactions of jets with inhomogeneous cloudy media, MNRAS, 359, 781. doi:10.1111/j.1365-2966.2005.08962.x

[22] Scott, J. H., Holman, G. D., Ionson, J. A., and Papadopoulos, K., 1980, The heating of gas in clusters of galaxies by relativistic electrons - collective effects, Ap.J., 239, 769.

[23] Su, M., and Finkbeiner, D. P., 2012, Evidence for gamma-ray jets in the Milky Way, Ap.J., 753, 61. doi:10.1088/0004-637X/753/1/61

[24] Zanni, C. et al., 2005, Heating groups and clusters of galaxies: the role of AGN jets, Astron. Astrophys., 429, 399.

\section{DISCUSSION}

MANEL PERUCHO Could you comment on the viscosity of the bow shock in your 3-D simulations? What is causing it?

JIM BEALL The viscosity is likely due to turbulence generated by the jet-ambient medium interaction and from internal shock structures within the jet and in the jet interface with the interstellar medium.

GENNADY BISNOVATYI-KOGAN Was a magnetic field included in your numerical simulations?

JIM BEALL The VH-1 code we are using is a hydrodynamic code with no capability for the inclusion of magnetic fields. We can estimate the magnetic field strength by assuming equipartition with the gas pressure, but this is not entirely satisfactory. We want to explore the PLUTO code, which has an MHD capability, in the near future. 